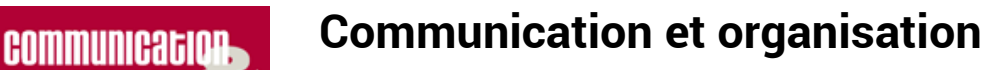

Or

Repenser la communication dans les organisations

publiques

\title{
Entretien avec Bernard Emsellem
}

\section{(2) OpenEdition}

\section{Journals}

Édition électronique

URL : http://journals.openedition.org/communicationorganisation/866

DOI : 10.4000/communicationorganisation.866

ISSN : $1775-3546$

Éditeur

Presses universitaires de Bordeaux

Édition imprimée

Date de publication : 1 juin 2009

Pagination : 242-244

ISSN : 1168-5549

Référence électronique

"Entretien avec Bernard Emsellem », Communication et organisation [En ligne], 35 | 2009, mis en ligne le 01 décembre 2012, consulté le 14 septembre 2020. URL : http://journals.openedition.org/ communicationorganisation/866 


\section{Entretien avec Bernard Emsellem}

Bernard Emsellem, 66 ans, titulaire d'une maîtrise de mathématiques pures et d'un DEA de logique mathématique, a commencé sa carrière par une thèse en sociologie de l'éducation sur les conditions de l'enseignement des maths à l'Institut de formation. Envoyé dans ce cadre par l'Unesco en Côte d'Ivoire, il y découvre l'ethnologie et en a gardé une passion pour l'art africain. " J'ai compris là-bas l'importance d'écouter les gens au-delà des seules statistiques ». À son retour, il devient conseil en stratégie marketing et directeur du département d'études qualitatives à la SEMA (1974-1981) puis à SOFRES Communication (1981-1985). Il crée son propre cabinet de conseil puis intègre le groupe de communication FRANCOM (19901995) en tant que directeur associé. En 1995, il devient directeur des stratégies puis président de TBWAlCorporate. En 2002, il rejoint la SNCF en tant que directeur de la communication. Il en est, depuis 2008, directeur général délégué au développement durable et à la communication. En 2009, Bernard Emsellem succède à Pierre Zémor, conseiller d'Etat honoraire, président fondateur, à la présidence de l'Association Communication publique, association des responsables de communication des institutions publiques. Il est par ailleurs viceprésident de la très puissante Union des Annonceurs (UDA).

Comment passe-t-on d'une agence, leader mondial de la communication corporate, comme TBWA à une entreprise publique comme la SNCF ?

Grâce à mes études antérieures, je me suis vite intéressé aux questions portant sur le destinataire. Il existe un écart notable entre ce que souhaite l'émetteur et ce qui est perçu par le destinataire. Dans le conseil, il y avait un devoir de trouver une réponse, aider à identifier le sujet et accompagner le client. Si c'est très intéressant et stimulant, cela ne permet pas toujours de pouvoir appréhender l'ensemble des facteurs qui conditionnent le processus de décision d'une organisation. Les interactions sont multiples, multi-rationnelles et empêtrées dans les systèmes d'interactions entre acteurs. Une bonne communication est un véritable levier pour que les interactions entre acteurs soient au service des objectifs collectifs.

Comment fabrique-t-on ses compétences pour devenir directeur de communication d'un grand groupe ?

Je dirais par hasard de glissements successifs. Si nous avons aujourd'hui la meilleure offre possible en France, c'est loin de donner le niveau nécessaire. La connaissance du terrain et l'expérience sont 
une véritable valeur ajoutée. Dans la communication, il y a une relation entre le destinataire et l'émetteur et c'est ce qui la différencie de l'information. Cette relation est le cœur même du travail de la communication. Les formations devraient prendre en compte davantage cet aspect, notamment grâce à la sociologie, qui permet de comprendre les comportements sociaux et la fabrication de communautés de travail et de cultures organisationnelles.

Quelle est la méthodologie de travail d'un directeur de communication de la SNCF ?

Notre méthodologie de travail se base sur une orientation client centrée. La communication n'est pas seulement le fait de travailler le client spécifiquement. Ceci est davantage le travail réalisé par le département marketing. Notre travail s'attache à saisir comment l'opinion publique nous perçoit et ce qu'elle pense de nous. L'intérêt est de diffuser les bonnes informations pour que nos clients partagent l'image que l'on veut diffuser de notre entreprise. Il s'agit de réduire l'écart entre l'image voulue et l'image perçue. Enfin, nous nous attachons à faire des opérations en externe qui soient l'occasion de créer des rencontres, afin de valoriser le client. Mais nous essayons aussi de multiplier les rencontres en interne. Il s'agit de faire vivre des relations durables entre l'organisation et ses publics pour assurer le terreau de la confiance.

\section{La SNCF, une entreprise nationale mais en concurrence ?}

C'est un fait, il y a de la concurrence. Mais dans la stratégie de la SNCF, je défends une posture de fierté et de pugnacité. Il est mieux de proposer une situation offensive par rapport à une situation d' « être là ». Le passé est une stratégie d'être là de la SNCF. Si notre histoire donne des fondements à cette fierté, c'est résolument vers l'avenir et l'innovation qu'on se positionne face à la concurrence. Pour autant, s'il est question ici de communication corporate, il y a là une dimension clairement publique par rapport à la population. C'est une entreprise publique dont l'intérêt est de créer du lien social. La $\mathrm{SNCF}$ est là pour créer du lien social. Irriguer la France, voire l'Europe, de ses habitants et de ses ressources, c'est donner de la vie au territoire. De plus globalement, les entreprises les plus professionnelles dans leur communication corporate ont tendance à rejoindre les principes de communication publique sur certains points. Il est question ici de légitimité du service qu'elles rendent à des clients qui sont aussi usagers et citoyens. On ne peut jamais strictement séparer ces identités. 


\section{Entretiens}

\section{Comment la crise a-t-elle affecté vos stratégies communication ?}

La crise est d'une brutalité telle que les organisations ont eu tendance à plonger encore plus que d'habitude dans des mesures d'urgence. Il faut agir rapidement et se focaliser sur la survie de la société. On voit alors se développer une communication de la société très prise sur le court terme. Mais être sur le court terme ne donne aucun sens. Il faut considérer les salariés comme parties prenantes. C'est pour cela que j'ai défendu une posture différente face à la crise, en développant un axe qui est présent dans notre stratégie depuis longtemps. Pour moi, il est très important de donner du sens aux actions qui sont menées par l'entreprise. Cela rejoint l'idée de fierté et de reconnaissance du passé. Il faut parler de l'avenir avant tout, mais sans oublier son passé.

Vous avez le double titre de directeur du développement durable et de la communication : deux responsabilités différentes ?

Il est intéressant de voir comment elles peuvent refonder l'identité et l'utilité de l'entreprise. La communication donne au développement durable de la visibilité. Le concept d'éco-mobilité est aujourd'hui évident. Oui, il existe des échanges entre ces deux sphères de compétences. Si je sépare l'idée de développement durable de la communication, on doit bien voir que la première thématique a une fonction transversale à toutes les directions, à l'instar de la qualité. Elle est une direction qui fonctionne sur la rencontre des directions, des métiers mais aussi des autres organisations (institutions publiques, fournisseurs, grands comptes...) C'est une problématique qui nous pousse à nous redéfinir, à échanger, à innover et à créer du lien.

Au sein de votre direction de communication, quelles sont les compétences que vous aimeriez voir se développer?

Résolument, apprendre comment le destinataire de nos messages nous perçoit et nous considère. Nous disons très peu en communication que l'intérêt est de faire «bouger» l'autre. C'est-à-dire l'amener à changer ses pratiques et à se repositionner. Il faut donc bien appréhender la perception de nos messages. Sont-ils entendus, compris, acceptés, discutés? Mènent-ils à des changements de comportements? Les audits de la SNCF se font aujourd'hui en externe. L'audit est nécessaire en amont et en aval de chaque action, encore plus en temps de crise. L'enquête qualitative en situation de crise est encore plus essentielle. C'est un dispositif d'évaluation qui n'est pas du tout remis en cause bien au contraire. 